\title{
Vortex lattice and matching fields for a long superconducting wire
}

\author{
Pablo A. Venegas* \\ Physics Department, University of California, Santa Cruz, California 95064 \\ Edson Sardella \\ Departamento de Física, Universidade Estadual Paulista, Caixa Postal 473, 17033-360, Bauru-SP, Brazil
}

(Received 9 May 1997; revised manuscript received 6 November 1997)

\begin{abstract}
We investigate the flux penetration patterns and matching fields of a long cylindrical wire of circular cross section in the presence of an external magnetic field. For this study we write the London theory for a long cylinder both for the mixed and Meissner states, with boundary conditions appropriate for this geometry. Using the Monte Carlo simulated annealing method, the free energy of the mixed state is minimized with respect to the vortex position and we obtain the ground state of the vortex lattice for $N=3$ up to 18 vortices. The free energy of the Meissner and mixed states provides expressions for the matching fields. We find that, as in the case of samples of different geometry, the finite-size effect provokes a delay on the vortex penetration and a vortex accumulation in the center of the sample. The vortex patterns obtained are in good agreement with experimental results. [S0163-1829(98)03430-4]
\end{abstract}

\section{INTRODUCTION}

Modern technology has made it possible to fabricate superconducting samples of small size, like films of thickness less than the London penetration length, as well as superconducting wires of radii of the order of this length. This has aroused the interest to study again the geometrical or size effects in superconductors. The size problem was already studied a long time ago, ${ }^{1,2}$ but recently it has been reconsidered, and some studies of transport currents, magnetization, magnetic moment, reversibility lines, and flux penetration in finite-size samples have been made. ${ }^{2-10}$ In particular, the penetration of the magnetic flux in finite-size superconductors has attracted the attention of physicists in the last years due to the fact that in finite samples the geometrical effects can produce important modifications in the critical state and in the resulting vortex distribution. As an example, we can cite the results of Zeldov et al. ${ }^{7}$ in thin superconductor strips that have been found to cause a delay in the penetration of the vortex lines, and a vortex accumulation at the center of the sample due to the geometrical barrier effect, but other studies in this respect have been made. ${ }^{2}$ In this way, our main interest in this work is to study, specifically, the size effects in the vortex lattice and in the matching fields (the minimum field for a new vortex penetration) for an infinite superconducting wire with a circular cross section.

We use the London theory to study the formation of the vortex lattice in the mixed state of a long superconducting cylinder. The London theory is valid in the limit of low induction (fields well below the upper critical field $H_{c 2}$ ), where most of the experiments can be performed to observe the vortex lattice. This theory fails in the limit of the small length scale. This breakdown of the theory is based upon the fact that the finite size of the vortex cores is neglected. Thus, the London theory is not suitable to treat the self-energy of a single vortex line. However, what really matters in determining the shape of the ground state of the vortex lattice is the interaction energy between vortices on different sites. For an infinite sample, vortices interact with each other via a twobody potential. Nevertheless, if the surface is taken into account, additional terms that describe the interaction of the vortex with the surface come in. In this work we develop the theory to describe such interactions in a long cylinder. In addition, we use the London theory to determine the free energy of the Meissner state of a long superconducting wire. For the determination of the ground state of the vortex lattice, we use the Monte Carlo simulated annealing minimization method. We obtain the ground-state lattice pattern starting from an arbitrary configuration. This procedure is different than the one usually used where the free energy of the vortex lattice for several predetermined configurations is calculated and the lowest one is chosen.

The paper is outlined as follows. In Sec. II we determine the magnetic field of an arbitrary distribution of vortices in the mixed state and its corresponding induction (spatial average of the local magnetic field). In addition, we find both the London and Gibbs free energy. In Sec. III we repeat this calculation to the Meissner state. In Secs. IV and V we analyze the matching fields and the vortex lattice patterns.

\section{MIXED STATE}

In what follows, we develop a theory for the mixed state of a long superconducting wire. Our starting point is the London equation. This equation is obtained from the second Ginzburg-Landau equation by assuming that the superconducting order parameter is a constant throughout the whole space, that is, it neglects variations of the order parameter inside the vortex core. The London approximation is valid provided that the Ginzburg-Landau parameter $\kappa=\lambda / \xi \gg 1$; here $\xi$ is the coherence length and $\lambda$ is the London penetration length. In addition, the vortices, whose size is of the order of $\lambda$, may overlap, but not the vortex cores. In cylindrical polar coordinates $(r, \phi)$, the London equation for the local magnetic field $\mathbf{h}=h \mathbf{z}$ is given by 


$$
-\lambda^{2}\left(\frac{\partial^{2} h}{\partial r^{2}}+\frac{1}{r} \frac{\partial h}{\partial r}+\frac{1}{r^{2}} \frac{\partial^{2} h}{\partial \phi^{2}}\right)+h=\Phi_{0} \sum_{i} \delta\left(\mathbf{r}-\mathbf{r}_{i}\right)
$$

where $\Phi_{0}$ is the quantum flux, $\mathbf{r}_{i}$ is the position of the $i$ th vortex inside the cylinder, and $\delta(\mathbf{r})=\delta(x) \delta(y)$ is the twodimensional $\delta$ function. Here we are assuming that the vortices are straight lines. Therefore, a three-dimensional (3D) problem is reduced to a $2 \mathrm{D}$ one. We will solve this equation subject to the following boundary conditions:

$$
\begin{aligned}
& h(a, \phi)=H, \\
& \left(\frac{\partial h}{\partial \phi}\right)_{r=a}=0,
\end{aligned}
$$

where $a$ is the radius of the cylinder. The first condition assures that outside the sample the field is uniform and is precisely the external field $H$; the second one states that the perpendicular component of the current vanishes at the boundary $r=a$, that is, the Cooper pairs cannot jump out of the sample.

To solve Eq. (1), we use the Green's-function method. Assuming for the Green's function that the boundary conditions $G\left(a, \phi, r^{\prime}, \phi^{\prime}\right)=0, G\left(r, \phi, r^{\prime}, \phi^{\prime}\right)$ continuous at $r$ $=r^{\prime}, \partial G\left(r, \phi, r^{\prime}, \phi^{\prime}\right) / \partial r$ discontinuous at $r=r^{\prime}$, and assuming that both $G$ and $h$ are periodic in $\phi$, one obtains

$$
\begin{aligned}
h\left(r^{\prime}, \phi^{\prime}\right)= & \Phi_{0} \sum_{i} G\left(r_{i}, \phi_{i}, r^{\prime}, \phi^{\prime}\right) \\
& -H a \lambda^{2} \int_{0}^{2 \pi} d \phi\left(\frac{\partial G}{\partial r}\right)_{r=a} .
\end{aligned}
$$

To proceed, we need to solve the equation for the Green's function. The method we use to find this function is outlined in Ref. 11 except for the fact that there the Green's function is associated with the Poisson equation and boundary conditions are taken at infinity. One has

$$
G\left(r, \phi, r^{\prime}, \phi^{\prime}\right)=\frac{1}{2 \pi \lambda^{2}}\left[K_{0}\left(\left|\mathbf{r}-\mathbf{r}^{\prime}\right| / \lambda\right)-\sigma\left(r, \phi, r^{\prime}, \phi^{\prime}\right)\right]
$$

where

$$
\begin{aligned}
\sigma\left(r, \phi, r^{\prime}, \phi^{\prime}\right)= & \sum_{m=-\infty}^{+\infty} \cos \left[m\left(\phi-\phi^{\prime}\right)\right] \\
& \times \frac{K_{m}(a / \lambda)}{I_{m}(a / \lambda)} I_{m}(r / \lambda) I_{m}\left(r^{\prime} / \lambda\right),
\end{aligned}
$$

where $I_{m}$ and $K_{m}$ are the modified Bessel functions.

We are now in a position to find the local magnetic field. Substituting Eq. (4) into Eq. (3) and using the identity $I_{m}(x) K_{m}^{\prime}(x)-I_{m}^{\prime}(x) K_{m}(x)=-1 / x$ (where the primes on $I$ and $K$ stands for the first derivative with respect to $x$ ) to develop the second term for the field expression, one obtains

$$
\begin{aligned}
h(r, \phi)= & \frac{\Phi_{0}}{2 \pi \lambda^{2}} \sum_{i}\left[K_{0}\left(\left|\mathbf{r}-\mathbf{r}_{i}\right| / \lambda\right)-\sigma\left(r, \phi, r_{i}, \phi_{i}\right)\right] \\
& +H \frac{I_{0}(r / \lambda)}{I_{0}(a / \lambda)} .
\end{aligned}
$$

The London (Helmholtz in the thermodynamic context) free energy contains basically two contributions. One is the energy stored in the field and the other one is the kinetic energy of the supercurrents. The London free energy per unit length is

$$
\begin{gathered}
\frac{F}{L}=\frac{1}{8 \pi} \int_{0}^{a} \int_{0}^{2 \pi} d r d \phi r\left[h^{2}+\lambda^{2}\left(\frac{\partial h}{\partial r}\right)^{2}+\frac{\lambda^{2}}{r^{2}}\left(\frac{\partial h}{\partial \phi}\right)^{2}\right] \\
=\frac{\Phi_{0}}{8 \pi} \sum_{i} h\left(r_{i}, \phi_{i}\right)+\frac{H a \lambda^{2}}{8 \pi} \int_{0}^{2 \pi} d \phi\left(\frac{\partial h}{\partial r}\right)_{r=a},
\end{gathered}
$$

where on going from the first to the second line we have used the London equation (1) and the boundary conditions (2) and the periodicity of the field. Here $L$ is the length of the system.

Now, the London free energy can be evaluated by introducing Eq. (6) into Eq. (7). This yields,

$$
\begin{aligned}
\frac{F}{L}= & \left(\frac{\Phi_{0}}{4 \pi \lambda}\right)^{2}\left\{\sum_{i, j}\left[K_{0}\left(\left|\mathbf{r}_{i}-\mathbf{r}_{j}\right| / \lambda\right)-\sigma\left(r_{i}, \phi_{i}, r_{j}, \phi_{j}\right)\right]\right. \\
& \left.+\left(\frac{\tilde{H}}{2}\right)^{2} \frac{a}{\lambda} \frac{I_{1}(a / \lambda)}{I_{0}(a / \lambda)}\right\}
\end{aligned}
$$

where $\widetilde{H}=H /\left(\Phi_{0} / 4 \pi \lambda^{2}\right)$.

To obtain the equilibrium configuration of the vortex lattice, the Helmholtz free energy is not convenient because the calculations involve a fixed magnetic field $H$. Therefore, it is necessary to perform a Legendre transformation to obtain the Gibbs free energy. The Gibbs free energy (in units of volume) is given by

$$
\mathcal{G}=\mathcal{F}-\frac{B H}{4 \pi}
$$

where $\mathcal{F}=F / A L$, with $A=\pi a^{2}$, and $B$ is the induction that is the spatial average of the local magnetic field

$$
B=\frac{1}{A} \int d^{2} r h
$$

The evaluation of this integral is tedious but straightforward. We obtain

$$
B=\frac{N \Phi_{0}}{A}+\frac{2 \pi a \lambda H}{A} \frac{I_{1}(a / \lambda)}{I_{0}(a / \lambda)}-\frac{\Phi_{0}}{A} \frac{1}{I_{0}(a / \lambda)} \sum_{i} I_{0}\left(r_{i} / \lambda\right) .
$$

Finally, we obtain for the Gibbs free energy 


$$
\begin{aligned}
\mathcal{G}= & \left(\frac{\Phi_{0}}{4 \pi \lambda^{2}}\right)^{2} \frac{\lambda^{2}}{A}\left[N \ln \kappa+\sum_{i \neq j} K_{0}\left(\left|\mathbf{r}_{i}-\mathbf{r}_{j}\right| / \lambda\right)\right. \\
& -\sum_{i, j} \sigma\left(r_{i}, \phi_{i}, r_{j}, \phi_{j}\right)+\widetilde{H} \sum_{i}\left(\frac{I_{0}\left(r_{i} / \lambda\right)}{I_{0}(a / \lambda)}-1\right) \\
& \left.-\left(\frac{\widetilde{H}}{2}\right)^{2} \frac{a}{\lambda} \frac{I_{1}(a / \lambda)}{I_{0}(a / \lambda)}\right] .
\end{aligned}
$$

Notice that the first term is the vortex self-energy and the second one describes the repulsive interaction between the vortices (the bulk term). The third term describes the attractive interaction between the vortices and image vortices located outside the sample. The effect of this interaction is to push the vortices close to the surface. The argument of the fourth term represents the flux $\Phi / \Phi_{0}=\left[1-I_{0}(r) / I_{0}(a)\right]$. The first term of this argument represents the repulsive interaction between a vortex and the magnetic field that penetrates the sample surface, and it pushes the vortices to the center of the sample. The second term in this argument represents the vortex magnetic energy. The fifth term is the Meissner state energy (see the next section). The competition between the vortex-vortex image interaction and the interaction between the vortex and the surface field represents an energy barrier that the vortex has to overcome to be able to enter the sample. When the external field is below the matching value, the vortex-vortex image interaction is more important and a new vortex is not able to enter. When the field is increased up to or above the critical value, the vortex can overcome the surface energy barrier and enters into the sample. On the other hand, we want to point out that the bulk interaction is invariant under any translation, but this symmetry is no longer valid for the whole free energy because of the presence of the various interactions. However, the system is still invariant under any rotation because it depends only on the angle difference.

\section{MEISSNER STATE}

In the Meissner state, although we have no penetration of the vortex lines, we have penetration of the magnetic field near the surface. In a semi-infinite superconductor, for instance, the external field penetrates exponentially over a distance $\lambda$. For a superconductor with cylindrical geometry, the field inside the sample is given by the London equation

$$
-\lambda^{2}\left(\frac{\partial^{2} h}{\partial r^{2}}+\frac{1}{r} \frac{\partial h}{\partial r}\right)+h=0 .
$$

The boundary condition is

$$
h(a)=H \text {. }
$$

The solution for this equation with the appropriate boundary conditions is

$$
h(r)=H \frac{I_{0}(r / \lambda)}{I_{0}(a / \lambda)} .
$$

Using Eq. (7), the London free energy per unit length can be written as

$$
\frac{F}{L}=\left(\frac{\Phi_{0}}{4 \pi \lambda}\right)^{2}\left(\frac{\widetilde{H}}{2}\right)^{2} \frac{a}{\lambda} \frac{I_{1}(a / \lambda)}{I_{0}(a / \lambda)},
$$

which is the second term of Eq. (8). Notice that if $a \gg \lambda$, near the surface the field has an exponential behavior $h$ $\sim H(a / r) e^{(r-a) / \lambda}$, as in a semi-infinite superconductor.

Similar to Eq. (11), we find for the induction

$$
B=\frac{2 \pi a \lambda H}{A} \frac{I_{1}(a / \lambda)}{I_{0}(a / \lambda)},
$$

which is the second term of Eq. (11).

The Gibbs free energy can be calculated by substituting Eqs. (16) and (17) into Eq. (9). One has

$$
\mathcal{G}=-\left(\frac{\Phi_{0}}{4 \pi \lambda^{2}}\right)^{2} \frac{\lambda^{2}}{A}\left[\left(\frac{\widetilde{H}}{2}\right)^{2} \frac{a}{\lambda} \frac{I_{1}(a / \lambda)}{I_{0}(a / \lambda)}\right],
$$

which is the fourth term of Eq. (12). The previous results comprise the framework for the discussion of the superconducting properties of a long cylinder. In the following sections we examine the matching fields and the vortex lattice patterns.

\section{MATCHING FIELDS}

The lower critical field $H_{c 1}$, defined as the lowest external field strong enough to cause penetration of at least one vortex line, is determined assuming that at the phase transition from the Meissner state to the mixed state, the Gibbs free energy per unit length, i.e., $\mathcal{G A}$, has the same value. In what follows, $S$ stands for the mixed state and $M$ for the Meissner state. One has,

$$
A \mathcal{F}_{M}-\frac{A B_{M} H_{c 1}}{4 \pi}=A \mathcal{F}_{S}-\frac{A B_{S} H_{c 1}}{4 \pi}
$$

where $\left(A \mathcal{F}_{M}, A B_{M}\right)$ is given by Eqs. (16) and (17) and $\left(A \mathcal{F}_{S}, A B_{S}\right)$ by Eqs. (8) and (11), respectively. Introducing these equations into Eq. (19), we find for the lower critical field

$$
H_{c 1}=\frac{\Phi_{0}}{4 \pi \lambda^{2}}\left[\frac{\ln \kappa-\frac{K_{0}(a / \lambda)}{I_{0}(a / \lambda)}}{1-\frac{1}{I_{0}(a / \lambda)}}\right] .
$$

Here we have taken the center of the cylinder as the equilibrium position of a single vortex line in Eqs. (8) and (11). In this case, only the $m=0$ term survives in the sum for $\sigma$ [cf. Eq. (5)]. By taking the limit of $a \rightarrow \infty$ in Eq. (20), we recover the well-known result $H_{c 1}^{\infty}=\left(\Phi_{0} / 4 \pi \lambda^{2}\right) \ln \kappa$. In Fig. 1 we plot the difference $\Delta H=H_{c 1}-H_{c 1}^{\infty}$ in units of $\left(\Phi_{0} / 4 \pi \lambda^{2}\right)$, which shows that the smaller the values of $a$, the larger the value of the lower critical field. In this way, this result shows clearly that the size effect provokes a delay in the first flux penetration, as found in experiments on other finite systems of different geometry. ${ }^{2,6}$

As a consequence of the energy barrier generated by the finite size of the sample, we have a delay not only for the first vortex line penetration, but for the subsequent lines too. 


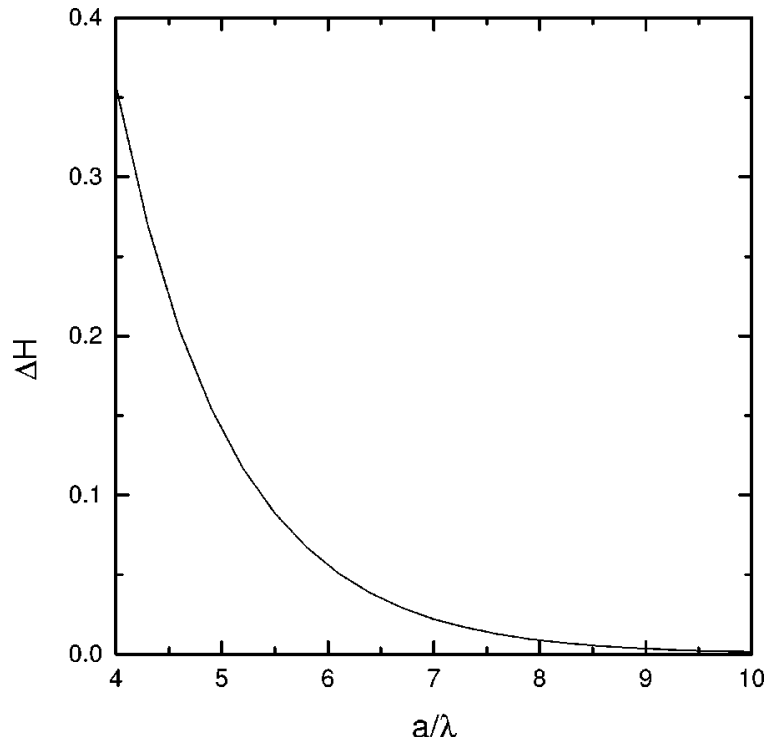

FIG. 1. The lower critical field difference $\Delta H=H_{c 1}-H_{c 1}^{\infty}$ in units of $\left(\Phi_{0} / 4 \pi \lambda^{2}\right)$ as a function of $a / \lambda$ for $\kappa=40$.

In this way, at low fields (near the lower critical field), we have a well-defined critical field for each new penetration (matching field) and, different from the bulk case, where the induction increases continuously with the external field, here the induction increases by steps as shown in Fig. 2. A new vortex enters the sample only when the energy is enough to overcome the surface energy barrier. As we go to higher magnetic fields, the induction approaches the bulk case.

The matching fields for each configuration of vortices $\left[\widetilde{H}_{s N}=H_{s N} /\left(\Phi_{0} / 4 \pi \lambda^{2}\right), N=1, \ldots, 18\right.$; with $\left.\widetilde{H}_{s 1} \equiv \widetilde{H}_{c 1}\right]$ are calculated using the same procedure used to obtain $\widetilde{H}_{c 1}$, i.e., equating the Gibbs free energy of the configurations with $N$ and $N+1$ vortices, $G_{N}=G_{N+1}$. In this way we obtain a tran-

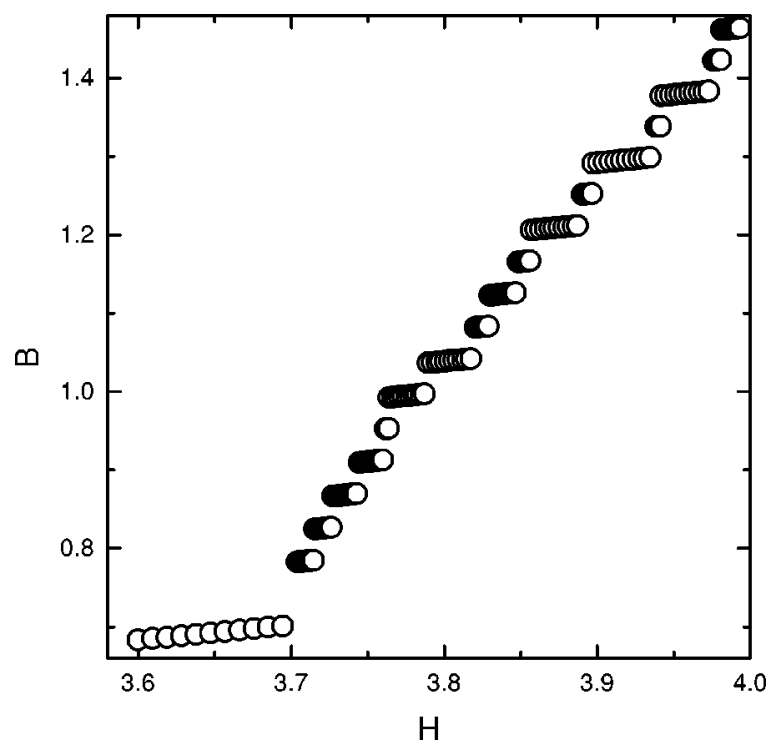

FIG. 2. Induction $(B)$ for $N=1$ to 18 vortices as a function of the external magnetic field $(H)$ in units of $\left(\Phi_{0} / 4 \pi \lambda^{2}\right)$. For each $N$ vortices configuration, the induction is calculated in the $\left[H_{s N}, H_{s N+1}\right]$ field range. Here we have used $a / \lambda=10, \lambda$ $=200 \mathrm{~nm}$, and $\kappa=40$.
TABLE I. Here $N_{0}$ represents a vortex at the center of the sample, $N_{1}$ and $N_{2}$ are the number of vortices on the first and second ring, and $R_{1}$ and $R_{2}$ are the respective radii of these rings. From 3 to 9 vortices, all the radii coincide up to the last digit shown in the table. Above 9 vortices, the internal radii $\left(R_{1}\right)$ coincide but, because of the fluctuations in the value of the external radii, we have assumed $R_{2}$ as the mean value. The seventh column is the energy associated with the terms that depend on the vortex positions and the last column represents the values of the critical fields for each configuration of $N$ vortices. The critical fields were determined with a precision of $10^{-7}$ and the energies of $10^{-10}$, respectively, though these two quantities are quoted with seven decimals. Here we have used the same parameters as in Figs. 2 and 3.

\begin{tabular}{lccccccc}
\hline \hline$N$ & $N_{0}$ & $N_{1}$ & $N_{2}$ & $R_{1}$ & $R_{2}$ & Energy & $H_{s N}$ \\
\hline 3 & & 3 & & 3.2745 & & 0.0348092 & 3.7047593 \\
4 & & 4 & & 3.6834 & & 0.0702077 & 3.7157228 \\
5 & & 5 & & 4.0392 & & 0.1283152 & 3.7313060 \\
6 & 1 & 5 & & 4.5206 & & 0.2092658 & 3.7468671 \\
7 & 1 & 6 & & 4.6694 & & 0.2931292 & 3.7489583 \\
8 & 1 & 7 & & 4.833 & & 0.4137502 & 3.7722957 \\
9 & 1 & 8 & & 4.999 & & 0.5784389 & 3.8003082 \\
10 & & 2 & 8 & 1.744 & 5.23 & 0.7557322 & 3.8088703 \\
11 & & 3 & 8 & 2.163 & 5.45 & 0.9599388 & 3.8258413 \\
12 & & 3 & 9 & 2.146 & 5.53 & 1.1768955 & 3.8335742 \\
13 & & 4 & 9 & 2.473 & 5.10 & 1.4435189 & 3.8639324 \\
14 & & 4 & 10 & 2.455 & 5.77 & 1.7204882 & 3.8702223 \\
15 & & 5 & 10 & 2.745 & 5.39 & 2.0593652 & 3.9075828 \\
16 & & 5 & 11 & 2.724 & 5.97 & 2.4020796 & 3.9106818 \\
17 & 1 & 5 & 11 & 3.178 & 5.60 & 2.7966109 & 3.9419102 \\
18 & 1 & 6 & 11 & 3.314 & 5.71 & 3.1954771 & 3.9465753 \\
\hline \hline
\end{tabular}

scendental equation in $\widetilde{H}$ (because the radii implicitly depends on $\widetilde{H}$ ) that can be solved iteratively. However, each time the free energy is calculated for a given magnetic field, this free energy must be calculated with the vortices in the equilibrium position. Thus, for each iteration the free energy must be minimized. The iterative work was performed using the Secant method and the minimization using the MonteCarlo simulated annealing method. ${ }^{14,15}$ For $a / \lambda=10$, the values obtained for $\widetilde{H}_{s N}$ can be seen in Table I.

\section{VORTEX LATTICES}

The usual procedure to find the ground state of the vortex lattice is to assume some particular geometry and then evaluate the Gibbs free energy. The configuration corresponding to the lowest value is supposedly, the most stable vortex lattice. Other authors ${ }^{12,13}$ have used the imaging method to determine the vortex configuration. In the present work we follow a different procedure. Using the Monte Carlo simulated annealing method, ${ }^{14,15}$ we start from an initial configuration chosen randomly, and we let the vortex lattice evolve towards the global minimum. The energy minimization is made using different initial configurations and different seeds for the random number generator. In this way we obtain different annealing schedules, ensuring that the system goes to the global minimum. This procedure has been usually avoided by many authors because the computational 
time greatly increases with the number of vortices. However, with the advent of very fast computers, this is no longer a major problem.

As the minimization method may require a large number of evaluations of the Gibbs free energy, we must find some effective manner of calculating $\sigma\left(r, \phi, r^{\prime}, \phi^{\prime}\right)$ [cf. Eq. (5)], which involves a sum of infinite terms. We will consider $a$ $\gg \lambda$, but still finite. Within this approximation, $I_{m}(a / \lambda)$ $\approx 1 / \sqrt{2 \pi a / \lambda} e^{a / \lambda}$ and $K_{m}(a / \lambda) \approx \sqrt{\pi / 2 a / \lambda} e^{-a / \lambda}$. One has,

$$
\sigma\left(r, \phi, r^{\prime}, \phi^{\prime}\right)=\pi e^{-2 a / \lambda} I_{0}\left(\left|\mathbf{r}+\mathbf{r}^{\prime}\right| / \lambda\right),
$$

where we have used the following identity: ${ }^{16}$

$$
\sum_{m=-\infty}^{+\infty} \cos (m \phi) I_{m}(x) I_{m}(y)=I_{0}\left(\sqrt{x^{2}+y^{2}+2 x y \cos \phi}\right) .
$$

The Gibbs free energy can be simplified to

$$
\begin{aligned}
\mathcal{G}= & \left(\frac{\Phi_{0}}{4 \pi \lambda^{2}}\right)^{2} \frac{\lambda^{2}}{A}\left[N \ln \kappa+\sum_{i \neq j} K_{0}\left(\left|\mathbf{r}_{i}-\mathbf{r}_{j}\right| / \lambda\right)\right. \\
& -\pi e^{-2 a / \lambda} \sum_{i, j} I_{0}\left(\left|\mathbf{r}_{i}-\overline{\mathbf{r}}_{j}\right| / \lambda\right)+\widetilde{H} \sum_{i}\left(\frac{I_{0}\left(r_{i} / \lambda\right)}{I_{0}(a / \lambda)}-1\right) \\
& \left.-\left(\frac{\widetilde{H}}{2}\right)^{2} \frac{a}{\lambda} \frac{I_{1}(a / \lambda)}{I_{0}(a / \lambda)}\right]
\end{aligned}
$$

where $\overline{\mathbf{r}}_{i}=-\mathbf{r}_{i}$.

To analyze the vortex patterns for each number of vortices we choose arbitrarily the magnetic field in the middle of the interval between $\widetilde{H}_{S N}$ and $\widetilde{H}_{S N+1}$. The minimization of the free energy was performed again using the simulated annealing method. As we have observed in Sec. II, the system is invariant under any rotation. Therefore, we can fix one of the vortices along the $x$ axis so that the minimization procedure will involve $2 N-1$ variables. We have done this for $N=3$ up to $N=18$. The vortex patterns for $N=3$ up to $N=11$ are illustrated in Fig. 3 and the complete numerical data for $N=3$ to 18 is shown in Table I. As can be seen from the figure and the table, the vortices arrange themselves in quite simple geometries. For example, for $N=3$, an equilateral triangle; for $N=4$, a square; for $N=5$, a pentagon; for $N=6$, a pentagon with a vortex at the center; for $N=7$, a hexagon with a vortex at the center; and for $N=18$, a vortex at the center, a first ring of six vortices forming a hexagon, and a second ring of eleven almost equally spaced vortices. The results show a clear tendency of the inner vortices to form a hexagonal lattice, however, the external vortices do not. For $N=3$ to 9 , the radii of all the vortices in the ring coincide, however, for $N>9$ the vortices in the outer ring show small radii fluctuations (no more than $7 \%$ ). For example, as we can see in Fig. 3 for the case of $N=10$, we have two vortices in a symmetric position related to the center of the sample and an external ring with 8 vortices. The vortices closer to the central ones have a bigger radius than those closer to the empty space between them. This is easy to understand based on the competition between the different
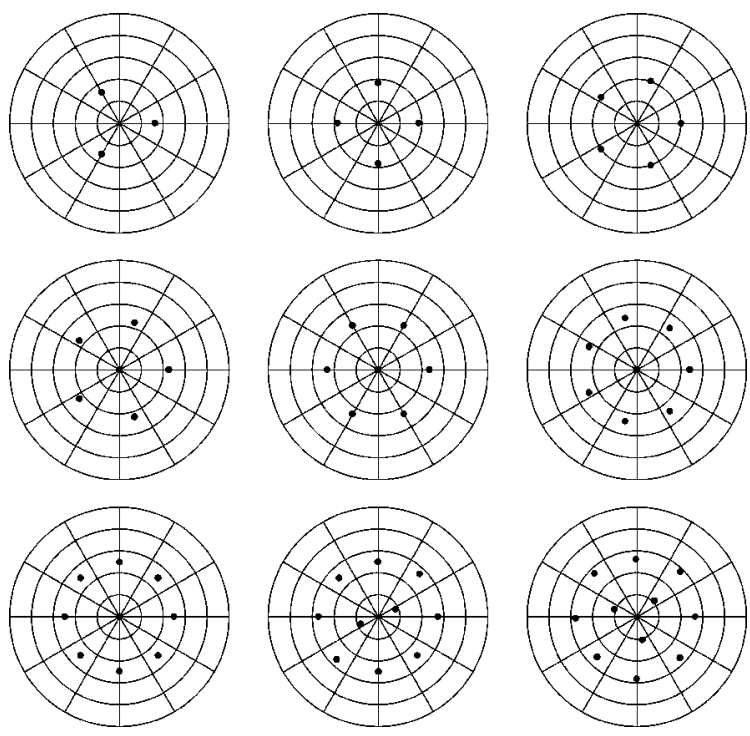

FIG. 3. Vortex patterns for different values for $N=3$ up to $N$ $=12$. The parameters used here are $a / \lambda=10$ and $\kappa=40$. The distance between adjacent circles is 2 (in units of $\lambda$ ).

interactions, as we pointed out at the end of Sec. II. When the external field is larger than $H_{s 10}$, the repulsive interaction of the vortices with the field that penetrates the surface is more important than the vortex-vortex image interaction. This interaction competes with the repulsive interaction between the vortices, which is more important between the central vortices and the vortices of the outer ring that are closer to them. This result agrees with the result of Ternovskii and Shekhata. ${ }^{17}$ They showed that for a semi-infinite plane, the vortex lattice is distorted near the surface.

As long as a decade ago, Yarmchuk and co-workers ${ }^{18}$ determined experimentally the vortex patterns up to 11 vortices in superfluids, and Campbell and Ziff ${ }^{19}$ made a very extensive numerical study of these patterns. The interaction potential between vortices in a superconductor runs over a distance $\lambda$, whereas in a superfluid this interaction is logarithmic and runs over all scales. Despite this difference in the interaction, the behavior of both systems is quite similar as explained below. The patterns found here are in excellent agreement with the experimental results found in Ref. 18 . The vortices not only show similar patterns, but show the same tendency to vortex accumulation at the center of the sample. The same tendency has been observed in experiments with finite-size superconducting samples of different geometries, ${ }^{2,6}$ and this suggests that this tendency is proper for finite samples independent of the particular geometry. Our results are also in good agreement with the results of Ref. 19, if we compare the ring structure, but two differences can be pointed out. First, as they supposed, the predetermined ring structures of their vortex patterns do not show the detailed structure of the outer rings as our more accurate calculations do. Second, their vortex patterns do not show the tendency of vortex accumulation at the center of the sample.

\section{SUMMARY}

In summary, by using the London theory we have studied the size effects on a long superconducting cylinder both in the mixed and Meissner states. We have determined numeri- 
cally the ground state of the system and have obtained the vortex patterns, the induction, and matching fields for $N$ $=3$ up to $N=18$ vortices. Our results show a clear tendency of vortex accumulation in the center of the sample, delay in the penetration of the flux lines, and irregularities in the distribution of the vortices closer to the surface, due to finitesize effects. Those effects have been found before in experiments with samples of different geometries showing,

*On leave from Departamento de Física, Universidade Estadual Paulista, Av. Engenheiro Luiz E. Coube S/N, 17033-360 BauruSP, Brazil.

${ }^{1}$ See, for example, E. Guyon, in Superconductivity, edited by P. R. Wallace (Gordon and Breach, New York, 1969), and references therein.

${ }^{2}$ E. H. Brandt, Rep. Prog. Phys. 58, 1465 (1995), and references therein.

${ }^{3}$ E. H. Brandt, Phys. Rev. B 46, 8628 (1992).

${ }^{4}$ E. H. Brandt and M. Indenbom, Phys. Rev. B 48, 12893 (1993).

${ }^{5}$ Th. Schuster, H. Kuhn, E. H. Brandt, and S. Klaumünzer, Phys. Rev. B 56, 3413 (1997).

${ }^{6}$ E. Zeldov, J. R. Clem, M. McElfresh, and M. Darwin, Phys. Rev. B 49, 9802 (1994).

${ }^{7}$ E. Zeldov, A. I. Larkin, V. B. Geshkenbein, M. Konczykowski, D. Majer, B. Khaykovich, V. M. Vinokur, and H. Shtrikman, Phys. Rev. Lett. 73, 1428 (1994).

${ }^{8}$ B. P. Thrane, C. Schlenker, J. Dumas, and R. Buder, Phys. Rev. B 54, 15518 (1996).

${ }^{9}$ L. Civale, T. K. Worthington, and A. Gupta, Phys. Rev. B 43, 5425 (1991). apparently, that this is a consequence of the finite size of the samples and not of the particular geometry.

\section{ACKNOWLEDGMENTS}

P.A.V. thanks the Brazilian Agency Capes for partial financial support. E.S. thanks the Brazilian Agencies CNPq and FAPESP for partial financial support. We thank Professor Mauro M. Doria for very useful discussions.

${ }^{10}$ B. Kaykovich, E. Zeldon, M. Konczykowsky, D. Majer, A. I. Larkin, and J. R. Clem, Physica C 235-240, 2757 (1994).

${ }^{11}$ J. D. Jackson, Classical Electrodynamics (Wiley, New York, 1962). See Section 3.10, page 84.

${ }^{12}$ A. Buzdin and D. Feinberg, Physica C 256, 303 (1996).

${ }^{13}$ S. H. Brongersma, E. Verweij, N. J. Koeman, D. G. de Groot, R. Griessen, and B. I. Ivlev, Phys. Rev. Lett. 71, 2319 (1993).

${ }^{14}$ W. H. Press, B. P. Flannery, S. A. Teukolsky, and W. T. Vetterling, Numerical Recipes (Cambridge University Press, Cambridge, England, 1992).

${ }^{15}$ S. Kirkpatrick, C. D. Gelatt Jr., and M. P. Vecchi, Science 220, 671 (1993).

${ }^{16}$ A. P. Prudinikov, Yu. A. Brychkov, and O. I. Marichev, Integrals and Series (Gordon and Breach, Amsterdam, 1986). See formula 5.910 .

${ }^{17}$ F. F. Ternovskii and L. N. Shekhata, Sov. Phys. JETP 35, 1202 (1972).

${ }^{18}$ E. J. Yarmchuk, M. J. V. Gordon, and R. E. Packard, Phys. Rev. Lett. 43, 214 (1979).

${ }^{19}$ L. J. Campbell and R. M. Ziff, Phys. Rev. B 20, 1886 (1979). 